\title{
Wachstumsbranche und Arbeitsplatzgenerator
}

\section{Werner Schneiter}

Dr. med., Kommunikationsberater Health Care und Mitglied der Puure-Huus Gruppe www.puure-huus.ch

Obwohl das schweizerische Gesundheitswesen als eines der besten der Welt gelten darf, stehen in den öffentlichen Debatten wegen steigender Gesundheitskosten vorwiegend die Aspekte des Systems im Vordergrund, die als problematisch wahrgenommen werden. Die Aktionsgruppe «Puure-Huus», eine Vereinigung freipraktizierender Ärzte, möchte dieser Tendenz etwas gegensteuern und auf die volkswirtschaftliche Bedeutung des Gesundheitswesens hinweisen.

\section{Arbeitsplätze}

Im Gesundheitswesen - eine der drei beschäftigungsintensivsten Branchen der Schweiz - sind heute 400000 Personen beschäftigt, was 300000 Vollzeitstellen entspricht. Durchschnittlich 70 Prozent dieser

Die Forderung nach Kosteneinsparungen ist unrealistisch, die Tatsachen werden verdrängt und verschleiert.

Personen sind Frauen. Im Gegensatz zu anderen Branchen, wo die Beschäftigtenzahlen stagnieren oder sinken, beträgt das jährliche Wachstum an Beschäftigten

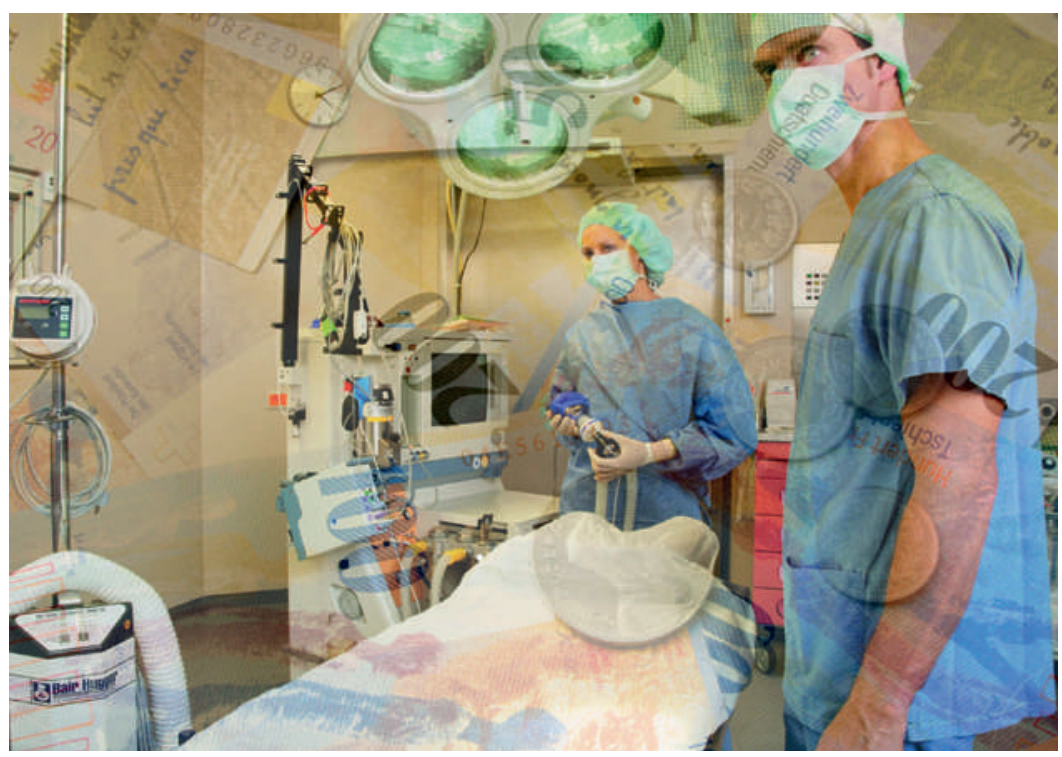

Das Schweizer Gesundheitswesen: gut und teuer. Aber es bringt auch mindestens so viel, wie es kostet. im Gesundheitswesen gemäss santésuisse 3 bis 5 Prozent oder 12000 bis 20000 Stellen. Bei einem durchschnittlichen Bruttoeinkommen von 70000 Franken pro Jahr ergibt dies eine Gesamtlohnsumme von rund 20 Milliarden Franken (die versteuert werden).

\section{Wertschöpfung}

Die Wertschöpfung der Branche betrug 2011 gemäss der CS-Studie von 20145 Prozent des BIP, stetig wachsend um geschätzte 0,5 Prozent jährlich. Gemessen am BIP der Schweiz von heute 700 Milliarden Franken, ergibt das einen Betrag von rund 50 Milliarden Franken.

Die volkswirtschaftliche Wirkung von Investitionen in Infrastruktur und Technik für Praxen, Heime, Spitäler, in Dienstleistungsorganisationen wie z.B. Spitex, die Leistungen der Industrie für Güter der Medizintechnik usw. sind dabei nicht berücksichtigt, weil Daten fehlen und sie nur schwer zu schätzen respektive zu berechnen ist.

\section{Gesundheitskosten}

Gesamthaft kostet die «Gesundheit der Schweiz» rund 70 Milliarden Franken. Fast ausschliesslich wird darüber wie folgt gesprochen, geschrieben, gesendet: «Die Kosten des Gesundheitswesens steigen weiter, in der Folge steigen die Krankenkassenprämien.» Das BAG und andere Organisationen mit eindeutiger politischer Ausrichtung prognostizieren dazu Prozentzahlen der zu erwartenden Prämienerhöhung - passend zu ihrer Strategie, den Leistungserbringern unablässig Spardruck zu machen. 
Die Kosten des Gesundheitswesens werden weiter steigen. Treiber dieser Kosten sind

- das Bevölkerungswachstum;

- die Demographie (mehr Ältere brauchen mehr Medizin, mehr Pflege);

- die steigenden Ansprüche mündiger Patientinnen und Patienten;

- der Einsatz von nichtmedizinischen Fachpersonen für das Gesundheitswesen;

- die wachsenden technologischen Möglichkeiten neuer Geräte und Systeme;

- neue Medikamente;

- die verkürzten Arbeitszeiten und die wachsenden Forderungen nach Teilzeitarbeit in den medizinischen Berufen;

- die hochspezialisierte Arbeitsteilung;

- die gesteigerten Bildungsvorschriften mit Zertifizierungen für Handlungserlaubnis;

- die Kultur des Misstrauens (Absicherung, Zweitmeinung, Arzt- und Klinikwechsel);

- der Angebotswettbewerb unter Leistungserbringern;

- die Mengenausweitung im ambulanten wie im stationären Leistungsbereich;

- zu tiefe Schwellen bei Franchise und Selbstbehalt.

Die Leistungserbringer sind dabei, von sich aus oder gezwungenermassen, Kosten einzusparen. Spitäler und Heime bewerkstelligen dies durch Straffung der Prozesse und Abläufe sowie - umstritten - Fallpauschalen (DRG); die Ärzte durch die relative Kürzung der Taxpunktwerte (Ärzteeinkommen sind seit 9 Jahren gleich geblieben) und den Einsatz von Generika; die Apotheker (und Ärzte) durch Margenreduktion; die Kassen durch striktere Kontrollen; alle durch den Einsatz von IT zur Entlastung der Administration. Sie alle strengen sich an, die Kostensteigerung zu bremsen oder zu stoppen. Sie alle haben bis heute schon wesentliche Beiträge zur Kostendämpfung geleistet.
Jedoch wird durch die kostentreibenden Entwicklungen in den oben genannten Feldern die Sparwirkung aufgehoben, sie wird überkompensiert.

Das schweizerische Gesundheitswesen ist gut und teuer. Um gut zu bleiben, werden die Kosten weiterhin steigen. Die Forderung nach Kosteneinsparungen von Politikern und Teilen der Gesundheitsökonomie ist unrealistisch, die Tatsachen werden verdrängt und verschleiert. Das ist nachvollziehbar, denn das Volk hat genug von schlechten Nachrichten, wer will schon seine Klientel verärgern. Andererseits ist erwiesen, dass die Schweizer ihr Gesundheitswesen als gut - die Kranken als sehr gut, die Gesunden als gut, aber teuer - beurteilen. «Man sollt' was tun, aber man kann nichts machen»wie man in Österreich zu sagen pflegt.

\section{Schlussfolgerung}

Der gesunde Menschenverstand würde die einfache Frage stellen: Was kostet es, was bringt es? Es ist an der Zeit, zur Kenntnis zu nehmen, dass das Gesundheitswesen der Volkswirtschaft mindestens das bringt, was es kostet, und dass wir mit diesen Kosten leben müssen. Politikerinnen, Politiker und andere, an einer breiten Wählerschaft Interessierte oder auf sie Angewiesene,

Es ist an der Zeit, zur Kenntnis zu nehmen, dass das Gesundheitswesen der Volkswirtschaft mindestens das bringt, was es kostet.

sowie die Leistungserbringer untereinander sollen endlich aufhören, den Schwarzen Peter einander zuzuschieben, Schuldige zu bezeichnen - es sind sowieso immer die anderen - und damit das Volk zu täuschen.

- Quellen: Studie CS 2014, Bundesamt für Statistik, santésuisse, USZ. 